\title{
The Classical Linearization Technique's Validity for Compliant Bearings
}

von Osmanski, Sebastian; Larsen, Jon S.; Santos, IImar F.

\section{Published in:}

Proceedings of the 10th International Conference on Rotor Dynamics (IFToMM 2018)

Link to article, DOI:

10.1007/978-3-319-99262-4_13

Publication date:

2018

Document Version

Peer reviewed version

Link back to DTU Orbit

Citation (APA):

von Osmanski, S., Larsen, J. S., \& Santos, I. F. (2018). The Classical Linearization Technique's Validity for Compliant Bearings. In K. Lucchesi Cavalca, \& H. Ingo Weber (Eds.), Proceedings of the 10th International Conference on Rotor Dynamics (IFTOMM 2018) (Vol. 1, pp. 177-191). Springer. Mechanisms and Machine Science Vol. 60 https://doi.org/10.1007/978-3-319-99262-4_13

\section{General rights}

Copyright and moral rights for the publications made accessible in the public portal are retained by the authors and/or other copyright owners and it is a condition of accessing publications that users recognise and abide by the legal requirements associated with these rights.

- Users may download and print one copy of any publication from the public portal for the purpose of private study or research.

- You may not further distribute the material or use it for any profit-making activity or commercial gain

- You may freely distribute the URL identifying the publication in the public portal 


\title{
The Classical Linearization Technique's Validity for Compliant Bearings
}

\author{
Sebastian von Osmanski ${ }^{1}$, Jon S. Larsen ${ }^{2}$, and Ilmar F. Santos ${ }^{3}$ \\ 1 Technical University of Denmark, Kgs. Lyngby, Denmark \\ 2 GEA Process Engineering A/S, Søborg, Denmark \\ 3 Technical University of Denmark, Kgs. Lyngby, Denmark, ifs@mek.dtu.dk
}

\begin{abstract}
The Gas Foil Bearing (GFB) is a promising and environmentally friendly technology allowing support of high-speed rotating machinery with low power loss and without oil or electronics. Unfortunately, GFBs provide limited damping, making an accurate prediction of the Onset Speed of Instability (OSI) critical. This has traditionally been assessed using linearised coefficients derived from the perturbed Reynolds Equation with compliance included implicitly. Recent work has, however, revealed significant discrepancies between OSIs predicted using these techniques and those observed from nonlinear analysis. In the present work, the perturbation method's underlying assumption on the pressure field is investigated by including the hitherto neglected pressurecompliance dependency directly. This leads to an extended perturbation akin to that commonly applied to tilting pad bearings and is shown to predict OSIs with much better agreement to time integration results. The extended perturbation method is cumbersome, but serves to highlight the error introduced when applying the classical perturbation method - as developed for rigid bearings by J. W. Lund - to GFBs.
\end{abstract}

Keywords: Gas Foil Bearings, Pertubation, Stability

\section{Introduction}

Due to the limited damping provided by GFBs, an accurate prediction of the Onset Speed of Instability (OSI) remains critical to their application. In order to predict the lateral vibration response of GFB-supported rotors, and hence their stability, it is possible to apply linear $[6,8-11,13,16,19,24]$ as well as nonlinear $[1-3,7,12,20]$ approaches. In the linear approach, the gas film forces are fundamentally represented by equivalent springs and dampers with coefficients derived from a linearisation of the Reynolds Equation (RE) around one or several states of equilibrium. The calculation of such gas film coefficients can be achieved by a numerical perturbation or analytically as proposed by Lund [17]. Peng and Carpino [19] were among the first to apply Lund's perturbation technique to GFBs and such analyses have since been performed by numerous authors $[6,10,24]$. Some of these have furthermore compared their results to nonlinear analyses showing varying levels of agreement. In recent work by the 
authors [14], this has been investigated by comparing the OSI of an industrial GFB-supported rotor predicted from the classical frequency domain technique to the stability limits observed from nonlinear time integration. Here, using equivalent numerical implementations for the two approaches, a significant discrepancy was demonstrated and shown to be increasing with the level of compliance.

A possible root cause of the observed disagreement can be found in a primary assumption of the classical perturbation approach, namely that the pressure field depends exclusively on the rotor position and velocity. Assuming subsequently the rotor to perform small harmonic oscillations, a Taylor series expansion can be inserted into RE to solve for the eight bearing stiffness and damping coefficients.

In the present work, the importance of the - thus far neglected - pressurecompliance dependency is investigated. The pressure field is thus assumed to depend not only on the rotor position and velocity, but also on the degrees of freedom (DOFs) representing the foil deflection. This additionally requires the solution of a dynamic pressure field for each of the foil DOFs and hence provides a coefficient matrix with contributions from each of these, analogous to the coefficient matrices often used for tilting pad journal bearings. Using a simple and widely studied GFB configuration [21] supporting a point mass as starting point, OSI predictions from the extended perturbation method are compared to results from both a classical perturbation method and a simultaneous nonlinear time integration. These two reference models are identical to those previously presented by the authors $[11,12,14]$ and have been experimentally validated. It should be emphasized that the vibrations occurring at the investigated OSI stems from a self-excited instability and thus are related exclusively to the homogeneous part of the equation system. This should not be confused with the onset of forced subsynchronous vibrations caused by the unbalance excitation in conjunction with the nonlinear GFB characteristics. The appearance of the latter is influenced by the level of unbalance, while the former is not.

The additional terms included in the extended perturbation multiplies the effort needed to attain the bearing coefficients and complicates their subsequent interpretation, but the extension is demonstrated to provide results in much better agreement to those obtainable from nonlinear time integration. The challenges of the extension are discussion and the significance of the foil-compliance terms is treated to illuminate the error introduced when neglecting them. In this sense, the novel contribution of the present work is to identify the limits of validity for the classical perturbation method with respect to compliance.

\section{The extended perturbation method}

In addition to the eccentricities, the pressure field $p$ is assumed to depend on $N$ DOFs. These dependencies can be collected in the vector $\mathbf{q}$ as

$$
\mathbf{q}=\left\{e_{x} e_{y} w_{1} \cdots w_{N}\right\}^{T} \in \mathbb{R}^{2+N},
$$

where $e_{x}$ and $e_{y}$ are the rotor eccentricity components and $w_{j}$ denotes the $j$-th foil compliance DOF. Using eq. (1), a first order Taylor series expansion of the 
pressure field around a state $\left(\mathbf{q}_{0}, \dot{\mathbf{q}}_{0}\right)$ can be written as

$$
p=p(\mathbf{q}, \dot{\mathbf{q}}) \approx p\left(\mathbf{q}_{0}, \dot{\mathbf{q}}_{0}\right)+\left.\frac{\partial p}{\partial \mathbf{q}}\right|_{\mathbf{q}_{0}, \dot{\mathbf{q}}_{0}}\left(\mathbf{q}-\mathbf{q}_{0}\right)+\left.\frac{\partial p}{\partial \dot{\mathbf{q}}}\right|_{\mathbf{q}_{0}, \dot{\mathbf{q}}_{0}}\left(\dot{\mathbf{q}}-\dot{\mathbf{q}}_{0}\right) .
$$

Defining $\mathbf{q}_{0}$ as the stationary point where $\dot{\mathbf{q}}=0$, and assuming all DOFs to exhibit small harmonic oscillations around this point with frequency $\omega_{s}$ as

$$
e_{\gamma}=e_{\gamma 0}+\Delta e_{\gamma} e^{i \omega_{s} t}, \gamma=x, y \quad \text { and } \quad w_{j}=w_{j 0}+\Delta w_{j} e^{i \omega_{s} t}, j=1, \ldots, N,
$$

the pressure field expansion from eq. (2) becomes

$$
p=p_{0}+p_{x} \Lambda_{x}+p_{y} \Lambda_{y}+\sum_{j=1}^{N} p_{w_{j}} \Lambda_{j},
$$

where

$$
\begin{aligned}
p_{0} & =p\left(\mathbf{q}_{0}\right), \\
p_{\gamma} & =\left(\left.\frac{\partial p}{\partial e_{\gamma}}\right|_{\mathbf{q}_{0}}+\left.i \omega_{s} \frac{\partial p}{\partial \dot{e}_{\gamma}}\right|_{\mathbf{q}_{0}}\right), \quad \gamma=x, y, \\
p_{w_{j}} & =\left(\left.\frac{\partial p}{\partial w_{j}}\right|_{\mathbf{q}_{0}}+\left.i \omega_{s} \frac{\partial p}{\partial \dot{w}_{j}}\right|_{\mathbf{q}_{0}}\right), \quad j=1, \ldots, N, \\
\Lambda_{\gamma} & =\Delta e_{\gamma} e^{i \omega_{s} t}, \quad \gamma=x, y, \\
\Lambda_{j} & =\Delta w_{j} e^{i \omega_{s} t}, \quad j=1, \ldots, N,
\end{aligned}
$$

so that $p_{0} \in \mathbb{R}$ and $p_{\gamma}, p_{w_{j}}, \Lambda_{\gamma}, \Lambda_{j} \in \mathbb{C}$.

\subsection{The film height function}

For the present purpose of investigating the pressure-compliance dependency, the rotor is assumed to be perfectly aligned and the foil is assumed to deform uniformly in the axial direction. Axial film height variations are hence neglected and the film height function becomes one-dimensional. This has previously been shown to be reasonable [23], but the proposed perturbation method is not restricted to such assumptions.

Referring to fig. 1b, the film height is composed of a rigid contribution,

$$
h_{r}=C+e_{x} \cos \theta+e_{y} \sin \theta,
$$

and a compliant contribution $h_{c}$ stemming from the deformation of the foil structure. It is hence necessary to establish a relation between the foil DOFs $w_{j}$ and the deformed shape of the top foil. The foil DOFs could be representing Fourier series amplitudes or polynomial coefficients, but for now they will represent discrete point deformations directly such that $w_{j}=h_{c}\left(\theta_{j}\right)$. To simplify the numerical implementation, the points $\theta_{j}$ are furthermore chosen to be coincident with the circumferential discretization of the fluid film. 


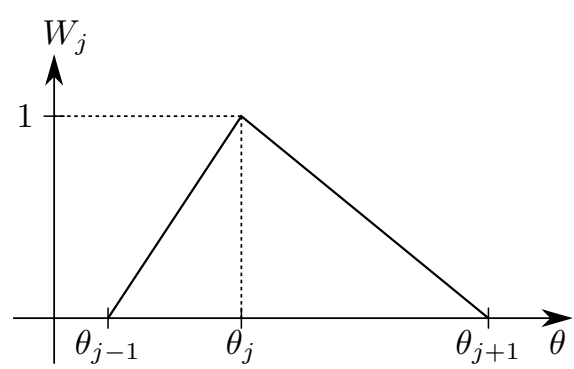

(a)

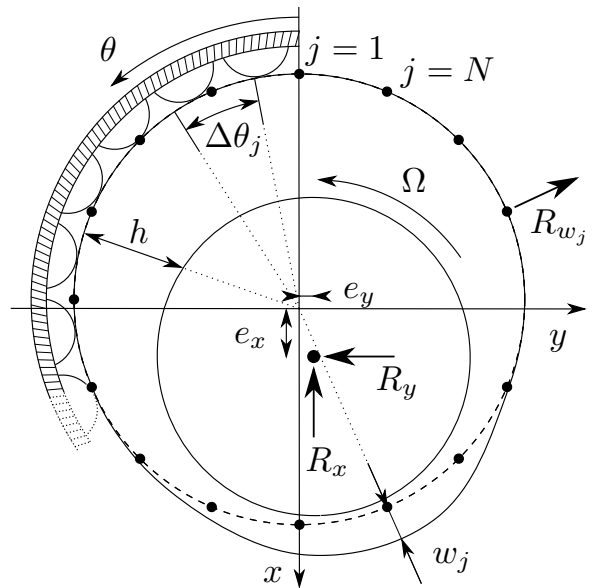

(b)

Fig. 1: (a) Weight function associated with the $j$-th foil compliance DOF; (b) Schematics of the GFB and illustration of the perturbed DOFs.

In order to evaluate the deformed shape between the discrete points, a simple linear interpolation is applied that is consistent with the linear shape functions applied for the finite element (FE) discretization of RE. A weight function is hence defined for each foil DOF as illustrated in fig. 1a and given as

$$
W_{j}(\theta)= \begin{cases}\frac{\theta-\theta_{j-1}}{\theta_{j}-\theta_{j-1}} & \text { if } \theta_{j-1}<\theta<\theta_{j} \\ \frac{\theta_{j+1}-\theta}{\theta_{j+1}-\theta_{j}} & \text { if } \theta_{j} \leq \theta<\theta_{j+1} \\ 0 & \text { otherwise, }\end{cases}
$$

such that the continuous compliant film height contribution can be written as

$$
h_{c}=\sum_{j=1}^{N} w_{j} W_{j}(\theta) .
$$

This finally allows, when introducing the perturbations from eq. (3), the perturbed film height to be written as

$$
h=h_{r}+h_{c}=h_{0}+\Lambda_{x} \cos \theta+\Lambda_{y} \sin \theta+\sum_{j=1}^{N} \Lambda_{j} W_{j}(\theta)
$$

where the static film height $h_{0}$ is given from

$$
h_{0}=C+e_{x 0} \cos \theta+e_{y 0} \sin \theta+\sum_{j=1}^{N} w_{j 0} W_{j}(\theta) .
$$




\subsection{Perturbation of Reynolds Equation}

Defining the circumferential and axial coordinates $\Theta=\theta R$ and $z$ in a bearing with radius $R$, the isothermal, compressible and transient RE can be written as

$$
\frac{\partial}{\partial \Theta}\left(\frac{p h^{3}}{12 \mu} \frac{\partial p}{\partial \Theta}\right)+\frac{\partial}{\partial z}\left(\frac{p h^{3}}{12 \mu} \frac{\partial p}{\partial z}\right)=\frac{\partial}{\partial \Theta}(p h U)+\frac{\partial}{\partial t}(p h),
$$

where $\mu$ is the gas viscosity and $U=R \Omega / 2$ is the circumferential gas film velocity. Substituting eqs. (4) and (13) into eq. (15) while neglecting higher order terms, the zeroth and first order equations can be separated. The zeroth order equation obtains the familiar form

$$
\frac{\partial}{\partial \Theta}\left(\frac{p_{0} h_{0}^{3}}{12 \mu} \frac{\partial p_{0}}{\partial \Theta}\right)+\frac{\partial}{\partial z}\left(\frac{p_{0} h_{0}^{3}}{12 \mu} \frac{\partial p_{0}}{\partial z}\right)=\frac{\partial}{\partial \Theta}\left(p_{0} h_{0} U\right)
$$

while the two first order equations for $p_{x}$ and $p_{y}$ can be written as

$$
\begin{array}{r}
\frac{\partial}{\partial \Theta}\left(\frac{p_{0} h_{0}^{3}}{12 \mu} \frac{\partial p_{\gamma}}{\partial \Theta}\right)+\frac{\partial}{\partial z}\left(\frac{p_{0} h_{0}^{3}}{12 \mu} \frac{\partial p_{\gamma}}{\partial z}\right)+\frac{\partial}{\partial \Theta}\left(\frac{p_{\gamma} h_{0}^{3}}{12 \mu} \frac{\partial p_{0}}{\partial \Theta}\right)+ \\
\frac{\partial}{\partial z}\left(\frac{p_{\gamma} h_{0}^{3}}{12 \mu} \frac{\partial p_{0}}{\partial z}\right)-\frac{\partial}{\partial \Theta}\left(p_{\gamma} h_{0} U\right)-i \omega_{s} p_{\gamma} h_{0}=-\frac{\partial}{\partial \Theta}\left(\frac{p_{0} h_{0}^{2} f_{\gamma}}{4 \mu} \frac{\partial p_{0}}{\partial \Theta}\right) \\
-\frac{\partial}{\partial z}\left(\frac{p_{0} h_{0}^{2} f_{\gamma}}{4 \mu} \frac{\partial p_{0}}{\partial z}\right)+\frac{\partial}{\partial \Theta}\left(p_{0} U f_{\gamma}\right)+i \omega_{s} p_{0} f_{\gamma}, \quad \gamma=x, y
\end{array}
$$

where $f_{x}=\cos \theta$ and $f_{y}=\sin \theta$, and, finally, the $N$ first order equations for $p_{w_{j}}$ related to the foil DOFs become

$$
\begin{array}{r}
\frac{\partial}{\partial \Theta}\left(\frac{p_{0} h_{0}^{3}}{12 \mu} \frac{\partial p_{w_{j}}}{\partial \Theta}\right)+\frac{\partial}{\partial z}\left(\frac{p_{0} h_{0}^{3}}{12 \mu} \frac{\partial p_{w_{j}}}{\partial z}\right)+\frac{\partial}{\partial \Theta}\left(\frac{p_{w_{j}} h_{0}^{3}}{12 \mu} \frac{\partial p_{0}}{\partial \Theta}\right)+ \\
\frac{\partial}{\partial z}\left(\frac{p_{w_{j}} h_{0}^{3}}{12 \mu} \frac{\partial p_{0}}{\partial z}\right)-\frac{\partial}{\partial \Theta}\left(p_{w_{j}} h_{0} U\right)-i \omega_{s} p_{w_{j}} h_{0}=-\frac{\partial}{\partial \Theta}\left(\frac{p_{0} h_{0}^{2} W_{j}}{4 \mu} \frac{\partial p_{0}}{\partial \Theta}\right) \\
-\frac{\partial}{\partial z}\left(\frac{p_{0} h_{0}^{2} W_{j}}{4 \mu} \frac{\partial p_{0}}{\partial z}\right)+\frac{\partial}{\partial \Theta}\left(p_{0} U W_{j}\right)+i \omega_{s} p_{0} W_{j}, \quad j=1, \ldots, N .
\end{array}
$$

\subsection{The coefficient matrix}

Having obtained $p_{0}, p_{\gamma}$ and $p_{w_{j}}$ by a suitable numerical solution scheme, these can be integrated to obtain the fluid film forces f. Using eq. (4), these can be written as

$$
\mathbf{f}=\left\{\begin{array}{c}
\int_{0}^{L} \int_{0}^{2 \pi}\left(p-p_{a}\right)\left\{\begin{array}{c}
\cos \theta \\
\sin \theta
\end{array}\right\} R d \theta d z \\
\int_{0}^{L} \int_{\theta_{1}-\frac{1}{2} \Delta \theta_{1}}^{\theta_{1}+\frac{1}{2} \Delta \theta_{1}}\left(p-p_{a}\right) R d \theta d z \\
\vdots \\
\int_{0}^{L} \int_{\theta_{N}-\frac{1}{2} \Delta \theta_{N}}^{\theta_{N}+\frac{1}{2} \Delta \theta_{N}}\left(p-p_{a}\right) R d \theta d z
\end{array}\right\}=\mathbf{f}_{0}+\left[\begin{array}{l}
\mathbf{Z}_{e e} \mathbf{Z}_{e w} \\
\mathbf{Z}_{w e} \mathbf{Z}_{w w}
\end{array}\right] \tilde{\mathbf{q}} e^{i \omega_{s} t}
$$


where the static force is given as the vector

$$
\mathbf{f}_{0}=\left\{\begin{array}{c}
\int_{0}^{L} \int_{0}^{2 \pi}\left(p_{0}-p_{a}\right)\left\{\begin{array}{c}
\cos \theta \\
\sin \theta
\end{array}\right\} R d \theta d z \\
\int_{0}^{L} \int_{\theta_{1}-\frac{1}{2} \Delta \theta_{1}}^{\theta_{1}+\frac{1}{2} \Delta \theta_{1}}\left(p_{0}-p_{a}\right) R d \theta d z \\
\vdots \\
\int_{0}^{L} \int_{\theta_{N}-\frac{1}{2} \Delta \theta_{N}}^{\theta_{N}+\frac{1}{2} \Delta \theta_{N}}\left(p_{0}-p_{a}\right) R d \theta d z
\end{array}\right\} \in \mathbb{R}^{2+N}
$$

and the dynamic forces are expressed in terms of the perturbation vector

$$
\tilde{\mathbf{q}}=\left\{\Delta e_{x} \Delta e_{y} \Delta w_{1} \cdots \Delta w_{N}\right\}^{T} \in \mathbb{R}^{2+N},
$$

along with the matrix $\mathbf{Z}$ with blocks given as

$$
\begin{aligned}
& \mathbf{Z}_{e e}=\int_{0}^{L} \int_{0}^{2 \pi}\left[\begin{array}{ll}
p_{x} \cos \theta & p_{y} \cos \theta \\
p_{x} \sin \theta & p_{y} \sin \theta
\end{array}\right] R d \theta d z \quad \in \mathbb{C}^{2 \times 2},
\end{aligned}
$$

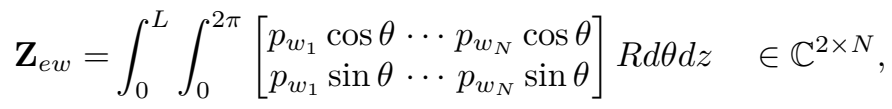

$$
\begin{aligned}
& \mathbf{Z}_{w e}=\left[\begin{array}{c}
\int_{0}^{L} \int_{\theta_{1}-\frac{1}{2} \Delta \theta_{1}}^{\theta_{1}+\frac{1}{2} \Delta \theta_{1}}\left[p_{x} p_{y}\right] R d \theta d z \\
\vdots \\
\int_{0}^{L} \int_{\theta_{N}-\frac{1}{2} \Delta \theta_{N}}^{\theta_{N}+\frac{1}{2} \Delta \theta_{N}}\left[p_{x} p_{y}\right] R d \theta d z
\end{array}\right] \in \mathbb{C}^{N \times 2}, \\
& \mathbf{Z}_{w w}=\left[\begin{array}{c}
\int_{0}^{L} \int_{\theta_{1}-\frac{1}{2} \Delta \theta_{1}}^{\theta_{1}+\frac{1}{2} \Delta \theta_{1}}\left[p_{w_{1}} \cdots p_{w_{N}}\right] R d \theta d z \\
\vdots \\
\int_{0}^{L} \int_{\theta_{N}-\frac{1}{2} \Delta \theta_{N}}^{\theta_{N}+\frac{1}{2} \Delta \theta_{N}}\left[p_{w_{1}} \cdots p_{w_{N}}\right] R d \theta d z
\end{array}\right] \in \mathbb{C}^{N \times N} .
\end{aligned}
$$

Finally, $\mathbf{Z}$ is related to the dynamic stiffness and damping coefficients as

$$
\begin{aligned}
& \mathbf{K}_{\text {fluid }}=\operatorname{Re}(\mathbf{Z})=\left[\begin{array}{ccccc}
k_{x x} & k_{x y} & k_{x w_{1}} & \cdots & k_{x w_{N}} \\
k_{y x} & k_{y y} & k_{y w_{1}} & \cdots & k_{y w_{N}} \\
k_{w_{1} x} & k_{w_{1} y} & k_{w_{1} w_{1}} & \cdots & k_{w_{1} w_{N}} \\
\vdots & \vdots & \vdots & \ddots & \vdots \\
k_{w_{N} x} & k_{w_{N} y} & k_{w_{N} w_{1}} & \cdots & k_{w_{N} w_{N}}
\end{array}\right] \quad \mathbb{R}^{(2+N) \times(2+N)}, \\
& \mathbf{D}_{\text {fluid }}=\frac{1}{\omega_{s}} \operatorname{Im}(\mathbf{Z})=\left[\begin{array}{ccccc}
d_{x x} & d_{x y} & d_{x w_{1}} & \cdots & d_{x w_{N}} \\
d_{y x} & d_{y y} & d_{y w_{1}} & \cdots & d_{y w_{N}} \\
d_{w_{1} x} & d_{w_{1} y} & d_{w_{1} w_{1}} & \cdots & d_{w_{1} w_{N}} \\
\vdots & \vdots & \vdots & \ddots & \vdots \\
d_{w_{N} x} & d_{w_{N} y} & d_{w_{N} w_{1}} & \cdots & d_{w_{N} w_{N}}
\end{array}\right] \quad \mathbb{R}^{(2+N) \times(2+N)}
\end{aligned}
$$

\subsection{Including the foil stiffness and damping}

The bearing coefficients in eqs. (26) and (27) are derived from the dynamic pressure fields and thus contain merely the contributions from the fluid film. 
When evaluating the displacement of the $j$-th foil DOF from its equilibrium $w_{j 0}$, the stiffness and damping of the foil itself should furthermore be considered.

Various elaborate foil structure models are available in the literature $[4,15$, $18,22]$, but the added complexity of these would not benefit the present comparison. Instead, the Simple Elastic Foundation Model (SEFM), as introduced by Heshmat et al [5], is employed with a uniform baseline stiffness derived from the widely applied expression by Walowit and Anno [25]

$$
k=\frac{E_{b}}{2 S_{b}}\left(\frac{t_{b}}{l_{0}}\right)^{3}\left(1-\nu_{b}^{2}\right)^{-1},
$$

where $E_{b}, S_{b}, t_{b}, l_{0}$ and $\nu_{b}$ are the bump foil's Young's modulus, pitch, thickness, half bump length and Poisson's ratio, respectively. The damping is assumed viscous and expressed simply as $d=k \eta / \omega_{s}$ where $\eta$ is a loss factor. A fundamental assumption of the SEFM is that all points in the foil behave independently, meaning that the foil structure is assumed not to contribute any cross couplings. Furthermore, the foil is not directly affecting the $e_{x}, e_{y}$ DOFs, meaning that the stiffness and damping matrices stemming from the bump foil can be written as

$$
\begin{aligned}
& \mathbf{K}_{\text {foil }}=L R k \operatorname{diag}\left[\begin{array}{lll}
0 & 0 & \Delta \theta_{1} \cdots \Delta \theta_{N}
\end{array}\right] \quad \in \mathbb{R}^{(2+N) \times(2+N)}, \\
& \mathbf{D}_{\text {foil }}=\eta \omega_{s}^{-1} \mathbf{K}_{\text {foil }} \quad \in \mathbb{R}^{(2+N) \times(2+N)},
\end{aligned}
$$

where $L$ is the axial length of the bearing and $\Delta \theta_{j}$ is the angular segment ascribed to the $j$-th foil DOF as illustrated in fig. $1 \mathrm{~b}$.

\subsection{The mass matrix}

The rotor is modelled as a point mass, implying that neither polar nor transverse inertia is considered. Combining the rotor mass with a rough estimate of the foil mass ascribed to each foil DOF, the system mass matrix is constructed as

$$
\mathbf{M}=\operatorname{diag}\left[m_{x} m_{y} m_{\text {foil }}^{\prime} R \Delta \theta_{1} \cdots m_{\text {foil }}^{\prime} R \Delta \theta_{N}\right] \in \mathbb{R}^{(2+N) \times(2+N)},
$$

where $m_{x}=m_{y}$ is the rotor mass and $m_{\text {foil }}^{\prime}$ is the average mass of the foil structure per unit circumferential length.

\subsection{System assembly}

Combining eqs. (26), (27), (29) and (30), the linearised $2+N$ equations of motion for the rotor-bearing system can be written as

$$
\mathbf{M} \ddot{\tilde{\mathbf{q}}}+\underbrace{\left(\mathbf{D}_{\text {foil }}+\mathbf{D}_{\text {fluid }}\right)}_{\equiv \mathbf{D}\left(\omega_{s}, \Omega\right)} \dot{\tilde{\mathbf{q}}}+\underbrace{\left(\mathbf{K}_{\text {foil }}+\mathbf{K}_{\text {fluid }}\right)}_{\equiv \mathbf{K}\left(\omega_{s}, \Omega\right)} \tilde{\mathbf{q}}=\mathbf{0},
$$

which can be recast into first order form using the state vector $\mathbf{z}=\{\tilde{\mathbf{q}} \dot{\tilde{\mathbf{q}}}\}^{T}$ as

$$
\dot{\mathbf{z}}=\underbrace{\left[\begin{array}{cc}
\mathbf{0} & \mathbf{I} \\
\mathbf{M}^{-1} \mathbf{K}\left(\omega_{s}, \Omega\right) & \mathbf{M}^{-1} \mathbf{D}\left(\omega_{s}, \Omega\right)
\end{array}\right]}_{\equiv \mathbf{A}\left(\omega_{s}, \Omega\right)} \mathbf{z},
$$

where $\mathbf{0}$ and $\mathbf{I}$ denote $(2+N) \times(2+N)$ zero and identity matrices, respectively. 


\section{Review of the classical two-DOF perturbation}

To emphasize the novel aspects of the proposed extended perturbation, a brief review of the traditional two-DOF approach employed with variations by e.g. $[6,8-11,13,16,19,24]$ is included. As originally introduced in the appendix of [17], a fundamental assumption is for the pressure field to be given as a function of rotor position and velocity only. This means that a Taylor expansion leads to a perturbed pressure field given as

$$
p=p_{0}+p_{x} \Lambda_{x}+p_{y} \Lambda_{y},
$$

which should be compared to eq. (4) of the extended perturbation. The foil compliance is then introduced implicitly through the film height function as

$$
h=C+e_{x} \cos \theta+e_{y} \sin \theta+f_{h_{c}}(p),
$$

where $f_{h_{c}}(p)$ is some function supplying the compliant film height as a function of pressure. For the SEFM incorporating a loss factor, this would be

$$
f_{h_{c}}(p)=k_{c}^{-1}\left(p-p_{a}\right) \quad \text { where } k_{c}=k(1+i \eta) .
$$

Inserting the film height eq. (34) and perturbed pressure eq. (33) into RE from eq. (15), equations for $p_{0}$ and $p_{\gamma}$ can be separated. The zeroth order equation is identical to the one obtained for the extended perturbation in eq. (16), while the first order equations for $p_{\gamma}$ become

$$
\begin{array}{r}
\frac{\partial}{\partial \Theta}\left(\frac{p_{0} h_{0}^{3}}{12 \mu} \frac{\partial p_{\gamma}}{\partial \Theta}\right)+\frac{\partial}{\partial z}\left(\frac{p_{0} h_{0}^{3}}{12 \mu} \frac{\partial p_{\gamma}}{\partial z}\right)+\frac{\partial}{\partial \Theta}\left(\frac{p_{\gamma} h_{0}^{2}\left(h_{0}+\left[3 p_{0} k_{c}^{-1}\right]\right)}{12 \mu} \frac{\partial p_{0}}{\partial \Theta}\right) \\
+\frac{\partial}{\partial z}\left(\frac{p_{\gamma} h_{0}^{2}\left(h_{0}+\left[3 p_{0} k_{c}^{-1}\right]\right)}{12 \mu} \frac{\partial p_{0}}{\partial z}\right)-\frac{\partial}{\partial \Theta}\left(p_{\gamma}\left(h_{0}+\left[p_{0} k_{c}^{-1}\right]\right)\right) U \\
-i \omega_{s} p_{\gamma}\left(h_{0}+\left[p_{0} k_{c}^{-1}\right]\right)=-\frac{\partial}{\partial \Theta}\left(\frac{p_{0} h_{0}^{2} f_{\gamma}}{4 \mu} \frac{\partial p_{0}}{\partial \Theta}\right) \\
-\frac{\partial}{\partial z}\left(\frac{p_{0} h_{0}^{2} f_{\gamma}}{4 \mu} \frac{\partial p_{0}}{\partial z}\right)+\frac{\partial}{\partial \Theta}\left(p_{0} U f_{\gamma}\right)+i \omega_{s} p_{0} f_{\gamma}, \quad \gamma=x, y
\end{array}
$$

where

$$
h_{0}=C+e_{x 0} \cos \theta+e_{y 0} \sin \theta+k_{c}^{-1}\left(p_{0}-p_{a}\right) .
$$

which should be compared to eq. (17) of the extended perturbation (the differences are framed in square brackets). The classical technique hence requires the solution of a nonlinear equation for $p_{0}$ and two linear complex equations for $p_{\gamma}$ from which the bearing coefficients can be extracted as

$$
\left[\begin{array}{ll}
k_{x x} & k_{x y} \\
k_{y x} & k_{y y}
\end{array}\right]+i \omega_{s}\left[\begin{array}{ll}
d_{x x} & d_{x y} \\
d_{y x} & d_{y y}
\end{array}\right]=\int_{0}^{L} \int_{0}^{2 \pi}\left[\begin{array}{lll}
p_{x} \cos \theta & p_{y} \cos \theta \\
p_{x} \sin \theta & p_{y} \sin \theta
\end{array}\right] R d \theta d z \quad \in \mathbb{C}^{2 \times 2}
$$

involving only the two rotor DOFs (per bearing). 


\section{Solution strategy}

The zeroth order eq. (16) is a nonlinear real equation in $p_{0}$ where $h_{0}$ is given from eq. (14). The assumption of an axially uniform deformation combined with the SEFM means that when solving for $p_{0}$, the discrete foil compliance $w_{j}$ is calculated in each iteration from the local mean axial static pressure as

$$
w_{j}=\frac{1}{k}\left(\frac{1}{L} \int_{0}^{L} p_{0}\left(\theta_{j}\right) d z-p_{a}\right) .
$$

The zeroth order equation is identical to that presented by the authors in [11] and the same solution strategy based on an FE discretization and Newton-Raphson iteration is employed. Notice that sub-ambient pressures are discarded when integrating the pressure fields (the Gümbel condition). As described in [11], this is meant to represent the effect of the top foil separation from the bump foil [5].

The $2+N$ first order eqs. (17) and (18) for $p_{\gamma}$ and $p_{w j}$ are linear complex equations which are solved using the FE discretization documented in [11]. Notice that while [11] treats the solution to the $p_{\gamma}$ equations produced by an implicit treatment of the foil compliance as in eq. (36), this collapses into eq. (17) for $k_{c}^{-1}=0$ and the same solution method can be applied.

A converged mesh of 17 axial (over half the bearing length, as symmetry is exploited) and 114 circumferential elements is used giving 2070 nodes. The number of circumferential locations, and hence the number of $p_{w_{j}}$ equation systems to be solved, is thus $N=115$.

\section{Results \& discussion}

The proposed method is evaluated by predicting the OSI for the simplest possible case of a point mass supported by a single-pad GFB in the widely studied configuration [21] listed in table 1. The OSI is calculated using: $(a)$ a simultaneous nonlinear time integration; $(b)$ a classical two-DOF perturbation; and $(c)$ the proposed extended perturbation. For the present bearing, eq. (28) predicts a foil stiffness of $4.6417 \mathrm{GN} / \mathrm{m}^{3}$ (case 2) which is evaluated along with a practically rigid (case 1: $4641.7 \mathrm{GN} / \mathrm{m}^{3}$ ) and a more flexible (case $3: 2.3209 \mathrm{GN} / \mathrm{m}^{3}$ ) variant. To put these stiffness levels into perspective, the maximum static foil deflection in the three cases at $20 \mathrm{kRPM}$ are $0.00,0.21$ and 0.40 times $C$ respectively.

\subsection{Stability analysis: Frequency domain}

To assess the linear stability limit of the rotor system described by eq. (32), a solution on the standard form $\tilde{\mathbf{z}}=\tilde{\mathbf{z}}_{0} e^{\lambda t}$ is substituted to produce the standard eigenvalue problem $\mathbf{A}\left(\omega_{s}, \Omega\right) \tilde{\mathbf{z}}=\lambda \tilde{\mathbf{z}}$. Having solved this, an eigenvalue $\lambda_{i}$ with positive real part then indicates the corresponding eigenmode $\tilde{\mathbf{z}}_{i}$ to be unstable.

Applying the classical two-DOF perturbation to a single bearing with coefficients given from eq. (38), four eigenvalues in complex conjugated pairs are 
Table 1: Properties of the GFB configuration, mainly from [21].

\begin{tabular}{|c|c|c|c|}
\hline $\begin{array}{l}\text { Journal } \\
\text { Load, } W_{x}=m_{x} g\end{array}$ & $30 \mathrm{~N}$ & Mass, $m=m_{x}=m_{y}$ & $3.059 \mathrm{~kg}$ \\
\hline \multicolumn{4}{|c|}{ Bearing configuration } \\
\hline Bearing radius, $R$ & $19.05 \mathrm{~mm}$ & Pad leading edge, $\theta_{l}$ & $0^{\circ}$ \\
\hline Bearing length, $L$ & $38.10 \mathrm{~mm}$ & Pad trailing edge, $\theta_{t}$ & $360^{\circ}$ \\
\hline Radial clearance, $C$ & $31.80 \mu \mathrm{m}$ & & \\
\hline \multicolumn{4}{|l|}{ Fluid properties } \\
\hline Ambient pressure, $p_{a}$ & $101.3 \mathrm{kPa}$ & Viscosity, $\mu$ & $1.950 \times 10^{5} \mathrm{Pas}$ \\
\hline \multicolumn{4}{|c|}{ Foil structure properties } \\
\hline Foil thickness, $t_{b}$ & $0.1016 \mathrm{~mm}$ & Bump pitch, $S_{b}$ & $4.572 \mathrm{~mm}$ \\
\hline Bump half length, $l_{0}$ & $1.778 \mathrm{~mm}$ & Bump height, $h_{b}$ & $0.5080 \mathrm{~mm}$ \\
\hline Young's modulus $E_{b}$ & $207.0 \mathrm{GPa}$ & Poisson's ratio, $\nu_{b}$ & 0.3 \\
\hline Density, $\rho_{b}$ & $8280 \mathrm{~kg} / \mathrm{m}^{3}$ & Loss factor, $\eta$ & 0.2 \\
\hline
\end{tabular}

obtained. By contrast, the extended perturbation in its current implementation and with the applied mesh results in $2(2+115)=234$ eigenvalues. As the eigenvalues of both methods depend on the oscillation frequency and the angular velocity, the stability limit, i.e. the OSI, is characterised by the condition

$$
\operatorname{Re}\left(\lambda_{i}\left(\omega_{s}, \Omega=\Omega_{\mathrm{OSI}}\right)\right)=0 \wedge \operatorname{Im}\left(\lambda_{i}\left(\omega_{s}, \Omega=\Omega_{\mathrm{OSI}}\right)\right)=\omega_{s},
$$

requiring the eigenvalue problem to be solved over a range of excitation frequencies and angular velocities. Here it should be highlighted that one should keep track of the eigenvalue order in between the $\left(\omega_{s}, \Omega\right)$ evaluation points. This is a trivial task for the two complex conjugated pairs arising from the two-DOF approach, but becomes a significant challenge for the 117 pairs obtained from the extended perturbation. At present, this has been handled by comparing the structure of the corresponding eigenvectors.

The result is shown in fig. 2, where the contours of the logarithmic decrement (LD) $\delta=-2 \pi \operatorname{Re}(\lambda) / \operatorname{Im}(\lambda)$ is plotted along with a single contour showing the concurrence of the excitation frequency $\omega_{s}$ and the damped natural frequency $\omega_{d}=\operatorname{Im}(\lambda)$. The crossing of the $\delta=0$ contour is exactly where eq. (40) is fulfilled and thus marks the OSI. The shown plot is for one of the two eigenmodes dominated by the rotor and is the one first becoming unstable. The mass of the foil from eq. (30) used in the eigenvalue solution is a rough estimate, but it has been verified that this has a vanishing influence on the OSI.

A similar plot is shown in fig. 3, but obtained using the traditional two-DOF perturbation method. The maps are similar, but the OSI is shifted more than $4 \mathrm{kRPM}$ and it is worth emphasizing the difference in computational cost. While both stability maps are based on solutions to 2805 eigenvalue problems, the extended perturbation requires solving $1172070 \times 2070$ complex linear equation systems to obtain the coefficients forming each of the $234 \times 234$ eigenvalue problems to be solved. In the traditional perturbation, each eigenvalue problem is 


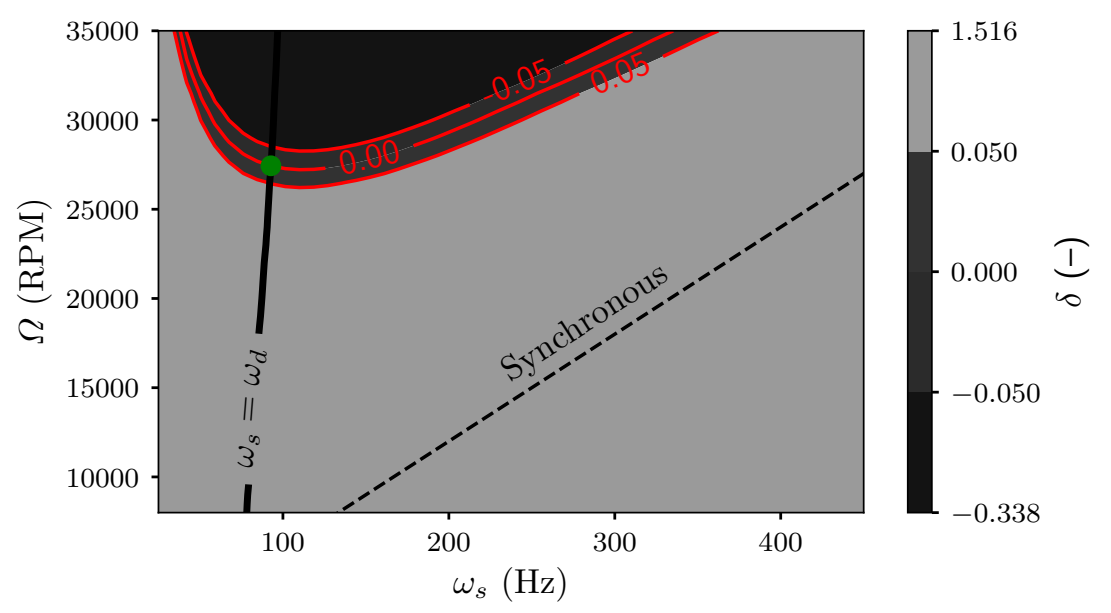

Fig. 2: Case 3 stability map based on the extended perturbation and eigenvalue solutions over a $51 \times 55\left(\omega_{s}, \Omega\right)$ grid. The OSI is marked at $(92.8 \mathrm{~Hz}, 27424 \mathrm{RPM})$.

merely $4 \times 4$ and the coefficients can be extracted from just two complex linear equation system solutions. The effort related to solving the zeroth order equation for each value of $\Omega$ is, however, the same for both methods. For the current implementation, the two-DOF perturbation calculations for fig. 3 take around $45 \mathrm{~s}$ while the extended perturbation calculations for fig. 2 take 10 times longer.

\subsection{Stability analysis: Time domain}

In the nonlinear sense, the stability limit is characterised by a breakdown of structural stability due to a small change in the angular velocity. At this point, the system Jacobian evaluated at the singular point representing the static equilibrium obtains an eigenvalue with zero real part. After this point, the behaviour will be governed by the system's nonlinear characteristics and should not be compared to the linear solutions.

To assess the stability limit, eq. (15) is FE discretized and simultaneously integrated in time with zero unbalance as described in [14]. The implementation has been carefully aligned to that used when solving the perturbed equations. A time integration can hence be started from the static equilibrium found from eq. (16) and the development of the oscillation amplitude can be observed. Such analysis is, however, subject to several challenges. First, even for an asymptotically stable state, the vibration amplitude of a numerical integration will never reach zero. Instead, it converges towards a noise floor specific to the numerical implementation. Initiating a time integration from a static equilibrium will hence always imply some oscillation. Second, even before the true structural stability limit is reached at $\Omega=\Omega_{\mathrm{OSI}}$, the static equilibrium state's basin of attraction will gradually narrow, increasing the possibility that an incidental numerical perturbation will push the system away from the stable equilibrium. 


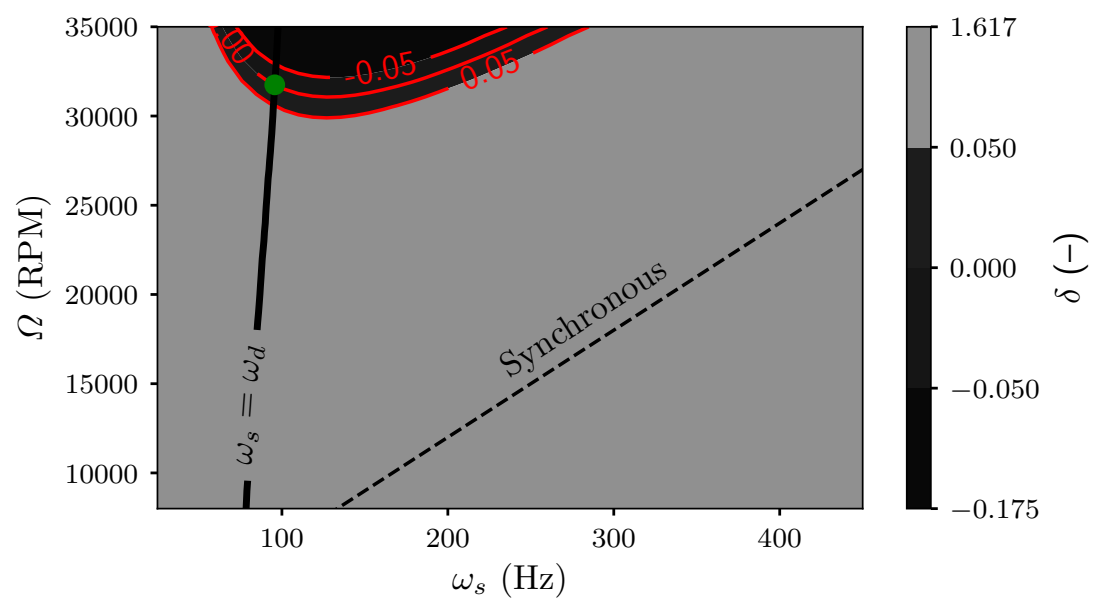

Fig. 3: Case 3 stability map based on the two-DOF perturbation and eigenvalue solutions over a $51 \times 55\left(\omega_{s}, \Omega\right)$ grid. The OSI is marked at $(95.7 \mathrm{~Hz}, 31769 \mathrm{RPM})$.

To demonstrate the uncertainty related to the OSI using time integration, $1 \mathrm{~s}$ simulations have been initialized from their static equilibria for every 10 RPM in the range 23-25 kRPM. For each response it is easy to judge whether the oscillation remains within the numerical noise level or grows exponentially. In fig. 4a, it is shown that some responses grow exponentially from around $23 \mathrm{kRPM}$, but that this is not persistent until $25.5 \mathrm{kRPM}$. This is, however, highly dependent on the tolerances applied in both the steady state solver and the subsequent time integration. More consistent results were found to be achievable when perturbing the static eccentricity just enough to leave the numerical noise floor. An initial perturbation of $10^{-10}$ on the eccentricity ratio $\left(=31.8 \times 10^{-16} \mathrm{~m}\right)$ thus results in tiny, but very clean oscillations from which the decay or growth can be identified. Plotting the LD obtained from curve fitting to each time series shows a monotonically decreasing curve with a distinct zero-crossing as shown in fig. $4 \mathrm{~b}$. The OSI identified using this method is denoted $\tilde{\Omega}_{\text {OSI }}$ to distinguish it from the more firmly defined OSI resulting from the frequency domain analysis.

\subsection{Comparison of OSIs}

The OSIs obtained using the perturbation methods and time integration are listed in table 2 for all three cases of foil stiffness. The excitation frequencies at the OSI are likewise listed for comparison. For the time integration, the latter has been extracted from frequency spectra based on $5 \mathrm{~s}$ simulations at angular frequencies slightly above $\tilde{\Omega}_{\text {OSI }}$. The relative OSI discrepancies are plotted in fig. 5 as a function of foil flexibility, and the extended perturbation is seen to provide results better in agreement with the nonlinear time integration results. 


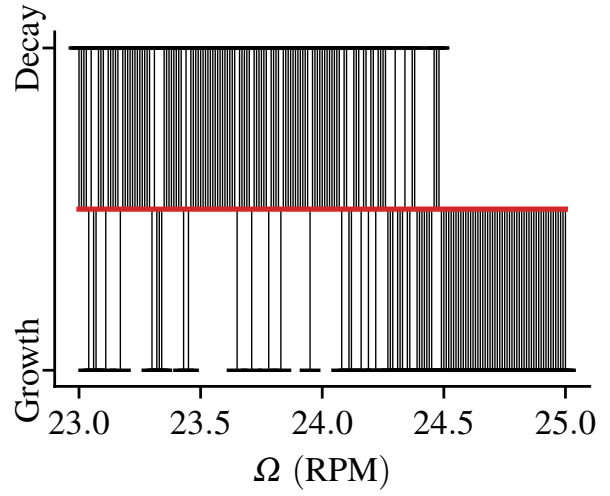

(a)

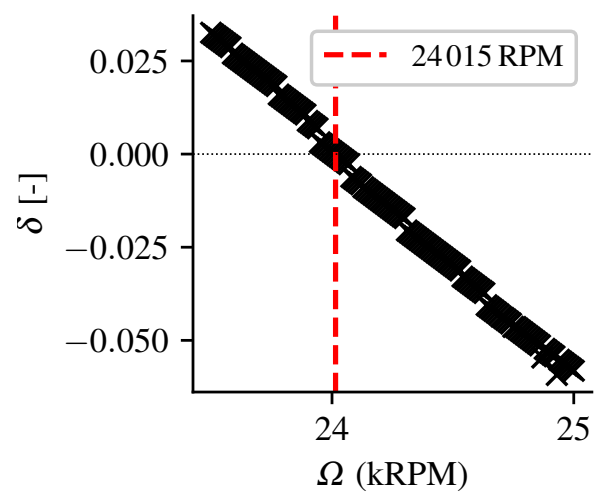

(b)

Fig. 4: (a) Stability of static equilibria judged from $1 \mathrm{~s}$ simulations for every 10 RPM (case 2); (b) LDs obtained by curve fitting to $0.25 \mathrm{~s}$ simulation after a rotor perturbation of $C \times 10^{-10}=31.8 \times 10^{-16} \mathrm{~m}$ for every $10 \mathrm{RPM}$ (case 2 ).

Table 2: Comparison of obtained OSIs

\begin{tabular}{cccccccc}
\hline \multirow{2}{*}{ Case } & $\begin{array}{c}\text { Foil stiffness } \\
k\left(\mathrm{GN} / \mathrm{m}^{3}\right)\end{array}$ & \multicolumn{2}{c}{ Time integration } & \multicolumn{2}{c}{ Two-DOF perturb. } & \multicolumn{2}{c}{ Extended perturb. } \\
& $k(\mathrm{RPM})$ & $\tilde{\omega}_{s}(\mathrm{~Hz})$ & $\Omega_{\mathrm{OSI}}(\mathrm{RPM})$ & $\omega_{s}(\mathrm{~Hz})$ & $\Omega_{\mathrm{OSI}}(\mathrm{RPM})$ & $\omega_{s}(\mathrm{~Hz})$ \\
\hline 1 & 4641.7 & 20868 & 112.8 & 21731 & 107.5 & 21731 & 107.5 \\
2 & 4.6417 & 24015 & 101.8 & 27868 & 103.4 & 24987 & 101.0 \\
3 & 2.3209 & 25118 & 91.7 & 31769 & 95.6 & 27424 & 92.8 \\
\hline
\end{tabular}

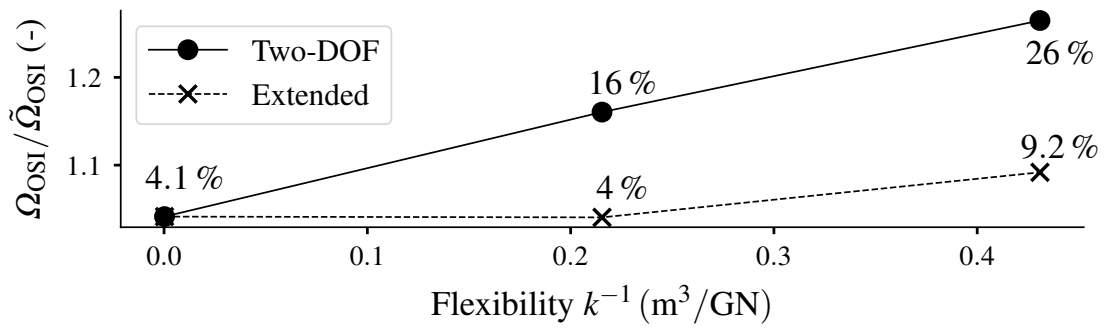

Fig. 5: Foil structure flexibility versus the discrepancy in the predicted OSI relative to the time integration result for both perturbation methods. 


\section{Conclusion}

Discrepancies in OSI between the traditional perturbation approach and time integration results were pointed out in [14], and this was postulated to be caused by a deficiency in the pressure expansion. This has been investigated in the present work by carrying through the perturbation method while including the additional pressure compliance terms in the Taylor expansion. In the presented form, these terms are treated straightforwardly using a high number of discrete foil deflections leading to a rather comprehensive model. This approach is debatable, as are the concrete choices of GFB properties and numerical schemes. The extended perturbation method has, however, been demonstrated to predict OSIs in much better agreement to time integration results, indicating the pressure-compliance terms to be significant.

Irrespective of the physical interpretation of the foil DOFs and their subsequent implementation, the fluid film coefficients affecting each foil DOF are eventually added to the contribution from the foil structure itself. The significance of the pressure-compliance terms will hence decrease for an increasingly stiff foil structure, matching the expectation for the two-DOF perturbation to be adequate for rigid bearings. This has also been demonstrated to be the case using the present implementation, as the methods converge for a very stiff foil.

When lowering the foil stiffness, the significance increases as the discrepancy of the two-DOF method grows and the two perturbation methods diverge. The baseline discrepancy of around $4 \%$ is ascribed to the determination of the time integration OSI, while the increased inconsistency of $9.2 \%$ for case 3 should be further investigated. The agreement is, however, still significantly better than the $26 \%$ discrepancy of the classical two-DOF method.

In conclusion, caution should be exercised if the classical perturbation method is applied to compliant bearings and the OSI of these should rather be assessed using either nonlinear methods or an extended perturbation as presented here.

\section{References}

1. Baum C, Hetzler H, Seemann W (2015) On the stability of balanced rigid rotors in air foil bearings. In: Proc. 11. Internationale Tagung Schwingungen in rotierenden Maschinen (SIRM2015), Magdeburg, Germany, pp 23-25

2. Bonello P, Pham HM (2014) The efficient computation of the nonlinear dynamic response of a foil-air bearing rotor system. J Sound Vib 333(15):34593478, DOI 10.1016/j.jsv.2014.03.001

3. Bonello P, Pham HM (2014) Nonlinear dynamic analysis of high speed oilfree turbomachinery with focus on stability and self-excited vibration. J Tribol 136(4):041,705, DOI 10.1115/1.4027859

4. Feng K, Kaneko S (2010) Analytical model of bump-type foil bearings using a link-spring structure and a finite-element shell model. J Tribol 132(2):021,706, DOI 10.1115/1.4001169

5. Heshmat H, Walowit JA, Pinkus O (1983) Analysis of gas-lubricated foil journal bearings. J Tribol Technol 105(4):647-655, DOI 10.1115/1.3254697 
6. Hoffmann R, Pronobis T, Liebich R (2014) The impact of modified corrugated bump structures on the rotor dynamic performance of gas foil bearings. In: Turbo Expo, ASME, p V07BT32A012, DOI 10.1115/GT2014-25636

7. Hoffmann R, Pronobis T, Liebich R (2015) Non-linear stability analysis of a modified gas foil bearing structure. In: Proc. 9th IFToMM Int. Conf. Rotor Dyn., pp 1259-1276, DOI 10.1007/978-3-319-06590-8_103

8. Kim TH, Andres LS (2008) Heavily loaded gas foil bearings: A model anchored to test data. J Eng Gas Turbine Power 130(1):012,504, DOI $10.1115 / 1.2770494$

9. Kim TH, San Andrés L (2006) Limits for high-speed operation of gas foil bearings. J Tribol 128(3):670-673, DOI 10.1115/1.2197851

10. Kim TH, San Andrés L (2007) Analysis of advanced gas foil bearings with piecewise linear elastic supports. Tribol Int 40(8):1239-1245, DOI 10.1016/ j.triboint.2007.01.022

11. Larsen JS, Santos IF (2014) Efficient solution of the non-linear reynolds equation for compressible fluid using the finite element method. J Braz Soc Mech Sci Eng 37(3):945-957, DOI 10.1007/s40430-014-0220-5

12. Larsen JS, Santos IF (2015) On the nonlinear steady-state response of rigid rotors supported by air foil bearings - theory and experiments. J Sound Vib 346:284-297, DOI 10.1016/j.jsv.2015.02.017

13. Larsen JS, Hansen AJ, Santos IF (2015) Experimental and theoretical analysis of a rigid rotor supported by air foil bearings. Mech Ind 16(1):106, DOI $10.1051 / \mathrm{meca} / 2014066$

14. Larsen JS, Santos IF, von Osmanski S (2016) Stability of rigid rotors supported by air foil bearings: Comparison of two fundamental approaches. J Sound Vib 381:179-191, DOI 10.1016/j.jsv.2016.06.022

15. Le Lez S, Arghir M, Frene J (2009) Nonlinear numerical prediction of gas foil bearing stability and unbalanced response. J Eng Gas Turbine Power 131(1):012,503, DOI 10.1115/1.2967481

16. Lee YB, Kim TH, Kim CH, Lee NS, Choi DH (2004) Unbalance response of a super-critical rotor supported by foil bearings - comparison with test results. Tribol Trans 47(1):54-60, DOI 10.1080/05698190490279038

17. Lund JW (1968) Calculation of stiffness and damping properties of gas bearings. J Tribol Technol 90(4):793-803, DOI 10.1115/1.3601723

18. von Osmanski S, Larsen JS, Santos IF (2017) A fully coupled air foil bearing model considering friction - theory \& experiment. J Sound Vib 400:660-679, DOI 10.1016/j.jsv.2017.04.008

19. Peng JP, Carpino M (1993) Calculation of stiffness and damping coefficients for elastically supported gas foil bearings. J Tribol 115(1):20-27, DOI 10. $1115 / 1.2920982$

20. Pham HM, Bonello P (2013) Efficient techniques for the computation of the nonlinear dynamics of a foil-air bearing rotor system. In: Turbo Expo, ASME, Texas, USA, DOI 10.1115/GT2013-94389

21. Ruscitto D, Mc Cormick J, Gray S (1978) Hydrodynamic air lubricated compliant surface bearing for an automotive gas turbine engine I - journal bearing performance. Tech. Rep. CR-135368, NASA, Cleveland, Ohio 
22. San Andrés L, Kim TH (2007) Improvements to the analysis of gas foil bearings: Integration of top foil $1 \mathrm{~d}$ and $2 \mathrm{~d}$ structural models. In: Turbo Expo, ASME, Montreal, Canada, vol 5, pp 779-789, DOI 10.1115/GT2007-27249

23. San Andrés L, Kim TH (2009) Analysis of gas foil bearings integrating FE top foil models. Tribol Int 42(1):111-120, DOI 10.1016/j.triboint.2008.05.003

24. Vleugels P, Waumans T, Peirs J, Al-Bender F, Reynaerts D (2006) Highspeed bearings for micro gas turbines: stability analysis of foil bearings. J Micromech Microeng 16(9):282-289, DOI 10.1088/0960-1317/16/9/S16

25. Walowit JA, Anno JN (1975) Modern developments in lubrication mechanics. Applied Science Publishers London 